\title{
Safety and Tolerability of Adjunctive Brivaracetam in Pediatric Patients < 16 Years with Epilepsy: An Open-Label Trial
}

\author{
Edwin Liu ${ }^{1} \cdot$ Deanne Dilley ${ }^{2,3} \cdot$ Belinda McDonough $^{4,5} \cdot$ Armel Stockis $^{6} \cdot$ Tony Daniels $^{2}$
}

Published online: 28 June 2019

(c) The Author(s) 2019

\begin{abstract}
Objective This trial evaluated the short-term safety and tolerability, steady-state pharmacokinetics, and preliminary efficacy of brivaracetam oral solution in children aged 1 month to $<16$ years with epilepsy.

Methods This was a phase IIa, open-label, single-arm, fixed three-step dose escalation trial of 3-weeks duration (N01263; NCT00422422). Patients were taking one to three concomitant antiepileptic drugs. Brivaracetam oral solution dosage, in two divided daily doses, was increased each week: approximately $0.8,1.6$, and $3.2 \mathrm{mg} / \mathrm{kg} /$ day for patients aged $\geq 8$ years, and $1.0,2.0$, and $4.0 \mathrm{mg} / \mathrm{kg} /$ day for patients aged $<8$ years.

Results Of the 100 patients enrolled, 90 (90.0\%) completed the trial. The safety population comprised 99 patients. Treatmentemergent adverse events (TEAEs) considered drug related by the investigator were reported by 32/99 (32.3\%) patients, most commonly ( $\geq 5 \%)$ somnolence $(7.1 \%)$ and decreased appetite $(6.1 \%)$. TEAEs were reported by $66 / 99(66.7 \%)$ patients, most commonly $(\geq 5 \%)$ convulsion, irritability, pyrexia, somnolence, and decreased appetite. In patients with a history of focal seizures with or without secondary generalization and no primary generalized seizures aged 4 to $<16$ years $(n=34)$, drugrelated TEAEs and TEAE incidences were $47.1 \%$ and $67.6 \%$, respectively. Steady-state trough brivaracetam and brivaracetam metabolite plasma concentrations increased proportionally with dose. The $\geq 50 \%$ responder rates (all seizure types) were $21.3 \%$ (all patients, $n=80$ ) and $36.4 \%$ (patients with focal seizures, aged 4 to $<16$ years, $n=22$ ).

Conclusions This open-label trial in pediatric patients with epilepsy provides preliminary information that short-term, adjunctive brivaracetam treatment is well tolerated and effective. Plasma concentrations of brivaracetam and metabolites increased with increasing dose.
\end{abstract}

Electronic supplementary material The online version of this article (https://doi.org/10.1007/s40272-019-00332-y) contains supplementary material, which is available to authorized users.

Edwin Liu

eliumd@ hotmail.com

1 Pediatric Neurologists of Palm Beach, 12959 Palms West Drive, Suite 120, Loxahatchee, FL 33470, USA

2 UCB Pharma, 8010 Arco Corporate Drive, Raleigh, NC 27617, USA

3 Statistics and Data Corporation, Tempe, AZ, USA

4 UCB Pharma, 208 Bath Road, Slough SL1 3WE, UK

5 MAFSAF Ltd, Belfast, Northern Ireland, UK

6 UCB Pharma, Chemin du Foriest, 1420 Braine l'Alleud, Belgium

\section{Key Points}

Short-term, add-on treatment with brivaracetam oral solution was generally well tolerated by children with epilepsy aged $<16$ years.

Plasma concentrations of brivaracetam and its metabolites increased proportionally with increasing dose.

This trial also provides preliminary evidence that addon brivaracetam treatment is effective in children with epilepsy.

\section{Introduction}

Despite the availability of new antiepileptic drugs (AEDs), $\geq 25 \%$ of pediatric patients with epilepsy have inadequate seizure control on currently available AEDs, or 
experience significant adverse drug effects [1]. Brivaracetam is a selective, high-affinity ligand for synaptic vesicle protein $2 \mathrm{~A}$ that demonstrated positive phase III results as adjunctive treatment for focal (partial-onset) seizures in adults with epilepsy [2-4]. Brivaracetam is indicated as adjunctive therapy and monotherapy for the treatment of focal (partial-onset) seizures in patients aged $\geq 4$ years (with injection indicated in those aged $\geq 16$ years) in the USA and as adjunctive therapy for focal (partial-onset) seizures with or without secondary generalization in patients aged $\geq 4$ years in the EU $[5,6]$ or in adults in other countries.

In healthy adults, brivaracetam is rapidly and completely absorbed, has a volume of distribution close to that of total body water, and has low plasma protein binding [7-9]. Brivaracetam is extensively biotransformed to three inactive metabolites by non-cytochrome P450 (CYP)-dependent hydrolysis of the acetamide group (acid metabolite [BRV$\mathrm{AC}]$; the primary pathway), CYP-dependent hydroxylation (hydroxy metabolite [BRV-OH]), and a combination pathway (hydroxy acid metabolite [BRV-OHAC]) [8, 10, 11]. The oral solution and film-coated tablet formulations have demonstrated bioequivalence in healthy adults [12].

The objectives of this trial were to assess the short-term safety and tolerability of adjunctive brivaracetam oral solution, to characterize the steady-state plasma concentrations of brivaracetam oral solution and its metabolites, and to obtain preliminary efficacy information in infants, children, and adolescents aged $<16$ years with epilepsy. An additional objective was to use the plasma concentration data to develop a population pharmacokinetic model to support dosing adaptations for children with epilepsy. This model has been published separately [13].

\section{Methods}

\subsection{Patients}

Patients aged $\geq 1$ month to $<16$ years, with a body weight $\geq 3 \mathrm{~kg}$, and with localized, generalized, or undetermined focal or generalized epileptic syndrome or other symptomatic generalized epilepsy, were included in the trial. The clinical diagnosis of epilepsy was supported using an electroencephalogram (EEG). Patients were taking at least one and no more than three concomitant AEDs (levetiracetam was prohibited), and had experienced one or more seizures during the 3 weeks before the trial. Patients were excluded if they had epilepsy secondary to a progressive cerebral disease, tumor, or any other progressive neurodegenerative disease; had status epilepticus in the month before screening or during the baseline period; had a history or presence of pseudoseizures; had febrile seizures only; or had previously received levetiracetam, unless discontinued at least 1 month before screening. Patients were also excluded if they had impaired hepatic function or had ever made a suicide attempt or had suicidal ideation during the previous 6 months as indicated by the Columbia-Suicide Severity Rating Scale completed at baseline. Pregnant or nursing females were not included.

To assess patients across a range of different age groups and with all types of epilepsy, patient enrollment was stratified by age category (infants and toddlers: 1 month to $<2$ years, at least 30 patients; children: 2 to $<12$ years, at least 30 patients; adolescents: 12 to $<16$ years, maximum 30 patients; a total of approximately 100 patients), and the number of patients with focal seizures was limited to $50 \%$ of the overall trial population.

\subsection{Trial Design}

This phase IIa, open-label, single-arm, fixed three-step doseescalation trial (N01263; NCT00422422) was conducted at 29 centers in the USA, Mexico, Belgium, Czech Republic, Poland, and Spain between July 2011 and March 2013. The trial comprised a 1-week baseline period and a 3-week evaluation period, followed by either entry into a long-term trial (N01266; NCT01364597) or a $\leq 2$-week downtitration period and a 2-week safety (post-treatment) period (Fig. 1).

Brivaracetam was administered as an oral solution (1 or $10 \mathrm{mg} / \mathrm{mL}$ ). Dosages administered in pediatric patients were based on the linear pharmacokinetic profile and effective dose range (50-200 $\mathrm{mg} /$ day) observed in adults, and scaling by body weight and age using physiologically based pharmacokinetic simulations (UCB data on file). During the evaluation period, the dose of brivaracetam per kilogram body weight was escalated based on age: patients aged $\geq 8$ years received approximately $0.8,1.6$, and $3.2 \mathrm{mg} / \mathrm{kg} /$ day in two divided daily doses at weeks 1,2 , and 3 , respectively; patients aged $<8$ years received approximately 1.0, 2.0, and $4.0 \mathrm{mg} / \mathrm{kg} /$ day in two divided daily doses at weeks 1 , 2 , and 3, respectively. Maximum doses were 50, 100, and $200 \mathrm{mg} /$ day at weeks 1,2 , and 3, respectively.

No additions or discontinuations of concomitant AEDs were permitted during the trial, and all concomitant AEDs were required to be at a stable dose $>7$ days before screening and throughout the trial. Benzodiazepines (if taken two or more times a week) and vagal nerve stimulators (if patients had a stable usage for $>2$ weeks before screening and throughout the trial) were considered concomitant AEDs. Felbamate was also prohibited unless patients had received it continuously for $>18$ months before screening.

The trial was conducted in accordance with the current version of the applicable regulatory and International Conference on Harmonization Good Clinical Practice requirements, the ethical principles as stated in the Declaration of Helsinki, and local laws of the countries involved. The 


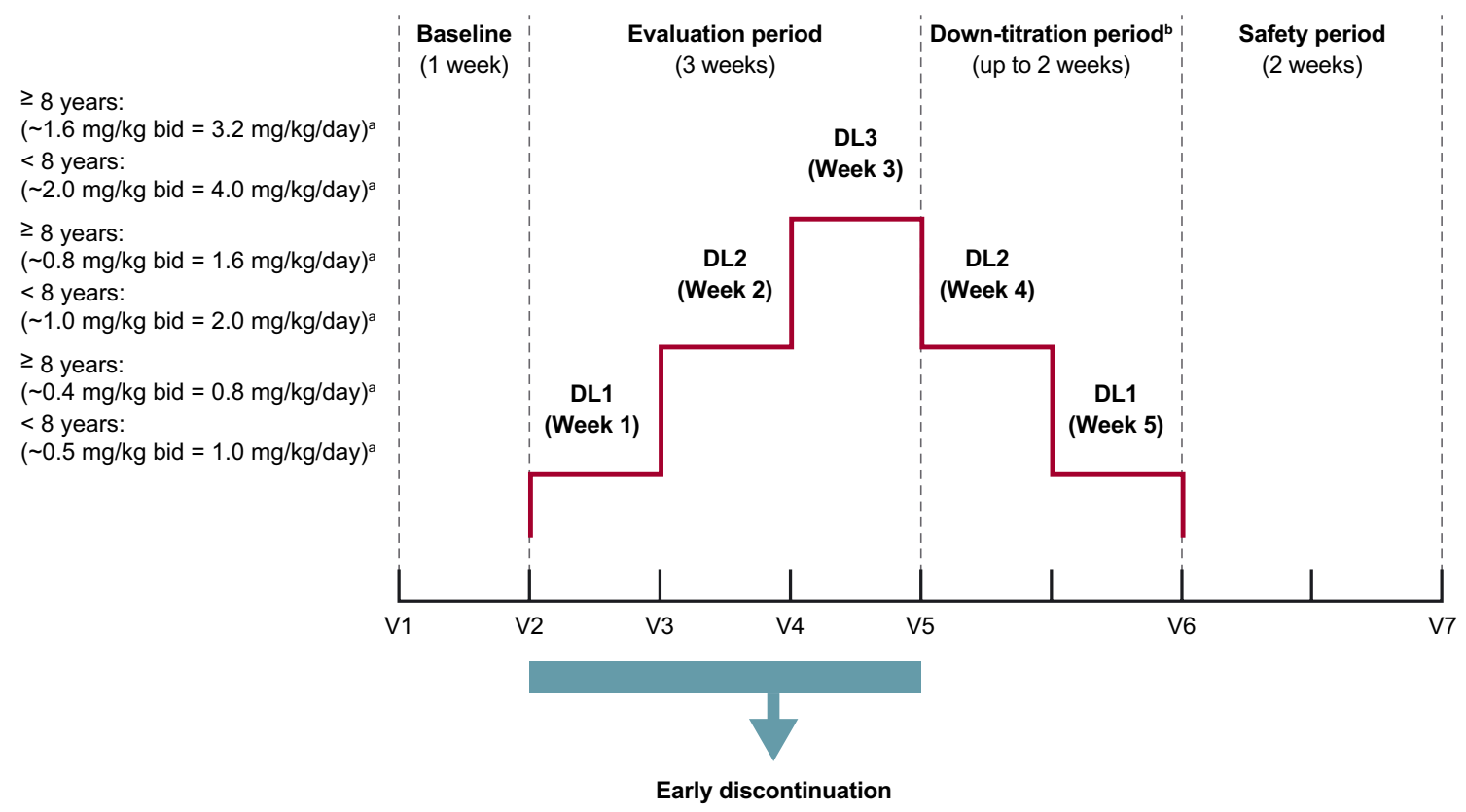

Fig. 1 Trial design. For V3, V4, and V5 (pharmacokinetic visits), there was a visit window of +2 days. For V2, V6, and V7, there was a visit window of \pm 2 days. ${ }^{\text {a }}$ Daily doses did not exceed 50,100, and

trial protocol, amendments, and patient informed consent form were reviewed by a national, regional, or independent ethics committee or institutional review board. A written consent form was signed and dated by the parent(s) or legal guardian(s) of the patients, and, where required, a consent form or a specific assent form was also signed and dated by the patients.

\subsection{Safety and Tolerability Assessments}

The safety and tolerability assessments performed throughout the trial were the occurrence of adverse events (AEs), laboratory tests, electrocardiograms (ECGs), body weight measurements, and monitoring of vital signs. AEs, coded using the Medical Dictionary for Regulatory Activities version 15.0, were summarized over the combined evaluation, downtitration, and safety periods. AEs were considered related to the trial drug if either the relationship to trial drug was specified as related by the investigator, or if the relationship to trial drug was not specified. Laboratory tests were conducted at baseline and following the high-dose treatment week (week 3). ECGs, monitoring of vital signs, and body weight measurements were carried out at baseline and the end of each trial week. Laboratory tests, ECGs, monitoring of vital signs, and body weight measurements were also carried out at a safety visit for patients not entering the longterm trial or 2 weeks after the last intake of trial drug for patients who discontinued early (see Fig. 1 for trial design). Clinically significant abnormal values for laboratory values
$200 \mathrm{mg}$ /day at weeks 1,2 , and 3 , respectively. ${ }^{\mathrm{b}}$ For patients who did not enter the long-term trial or discontinued early. bid twice daily, $D L$ dose level, $V$ visit

and vital signs were defined in the statistical analysis plan. The clinical significance of ECG results was determined by the investigator.

\subsection{Plasma Concentration of Brivaracetam and Metabolites}

At least two blood samples were obtained for determination of plasma concentrations of brivaracetam and metabolites at the end of the low-, mid-, and high-dose treatment weeks (weeks 1, 2, and 3), or at the early discontinuation visit. Blood samples were taken in any one of three possible time brackets (early morning, late morning, or afternoon). For early morning, one sample was taken immediately before the morning dose of brivaracetam and one at 1-2 $\mathrm{h}$ post-dose; for late morning, two samples were taken $2-6 \mathrm{~h}$ after the morning dose of brivaracetam, with $\geq 2 \mathrm{~h}$ between sampling times; for the afternoon, two samples were taken 6-12 $\mathrm{h}$ after the morning dose of brivaracetam, with $\geq 2 \mathrm{~h}$ between sampling times. Plasma concentrations of brivaracetam and metabolites (BRV-AC, BRV-OH, and BRV-OHAC) were determined using liquid chromatography with tandem mass spectrometry detection [13].

\subsection{Efficacy Assessments}

Seizures were recorded on daily record cards completed by the patient or parent/caregiver. Responder rate was calculated as the percentage of patients with $\geq 50 \%$ reduction in 
the number of seizure days (all seizure types) from the baseline period to the end of the evaluation period, standardized to a 28-day duration. Responder rate could therefore only be calculated in patients reporting at least one seizure during the baseline period. Exploratory efficacy variables were number of seizure days (all seizure types) over the evaluation period standardized to a 28 -day duration, absolute and percent reduction from baseline in number of seizure days (all seizure types) per 28 days during the evaluation period, and seizure freedom rate during the evaluation period, defined as patients who completed the evaluation period with no missing diary days and no reported seizures.

\subsection{Statistics}

The safety set (SS) comprised all enrolled patients who took at least one dose of brivaracetam. Safety and tolerability outcomes are presented for the SS for all patients and the subgroup with a history of focal seizures with or without secondary generalization and no primary generalized seizures (all patients aged $<16$ years, $<4$ years, and 4 to $<16$ years). The pharmacokinetic per-protocol set (PK-PPS) comprised all patients with at least one measurable plasma sample (with recorded sampling time) on at least one visit with documented drug intake times. Plasma concentration results are shown for patients with a history of focal seizures (corresponding to the approved indication) split in three age bands $(<4$ years, 4 to $<16$ years, $<16$ years). The full analysis set (FAS) comprised all patients in the SS, with baseline and at least one completed post-baseline daily record card or EEG. Efficacy outcomes are presented descriptively for the FAS by seizure history and age group.

No imputation of missing values was performed for analysis parameters. Imputations for missing or partial values for dates for AEs and concomitant medications were applied to determine whether an event was to be considered treatmentemergent or concomitant. Only reported data were used in each analysis time interval.

\section{Results}

\subsection{Patients}

Overall, 100 patients entered the trial; 99 patients were included in the SS (one patient did not take any trial drug and was excluded), 96 were included in the PK-PPS, and 97 were included in the FAS. Of the 100 patients enrolled, $90(90.0 \%)$ completed the trial and 10/100 (10.0\%) discontinued (AEs: 6/100 [6.0\%]; lack of efficacy: 1/100 [1.0\%]; protocol violation: $1 / 100$ [1.0\%]; consent withdrawn: $2 / 100$ [2.0\%]).
Baseline demographic and epilepsy characteristics are presented in Table 1. Overall, mean \pm standard deviation age was $6.3 \pm 4.8$ years and body weight was $24.2 \pm 16.2 \mathrm{~kg}$. Of the overall population, approximately one-third (34/99 [34.3\%]) were patients aged 4 to $<16$ years who had a history of focal seizures with or without secondary generalization and no primary generalized seizures. Among those, $22 / 34(64.7 \%)$ experienced focal seizures during the 1-week prospective baseline period. The median duration of brivaracetam exposure during the evaluation period was 22 days (range 1-29) for both the total population and the subgroup with focal seizures aged 4 to $<16$ years. Overall, 81/99 (81.8\%) patients were evaluated for 3-4 weeks.

This article focuses on the subpopulation of children aged $\geq 4$ years with focal seizures because, as detailed in the introduction, brivaracetam is currently indicated for the treatment of focal seizures in children aged $\geq 4$ years with epilepsy, and health authorities (the European Medicines Agency [EMA] and the US FDA) have determined that efficacy can be extrapolated from adults to children aged $\geq 4$ years $[14,15]$. Data for the stratification age categories ( 1 month to $<2$ years, 2 to $<12$ years, 12 to $<16$ years) are shown in the Electronic Supplementary Material (Tables S1-4 Fig. S1).

\subsection{Safety and Tolerability}

Throughout the trial, 66/99 (66.7\%) patients/caregivers reported treatment-emergent adverse events (TEAEs); in $32 / 99(32.3 \%)$ cases, TEAEs that the investigator considered related to trial drug were reported (Table 2). Most TEAEs were considered mild or moderate in intensity. Six of 99 (6.1\%) patients reported TEAEs leading to discontinuation, all of which were considered by the investigator to be related to trial drug. Two patients, both with a history of behavioral disturbances, discontinued brivaracetam due to aggression. One patient with a history of tuberous sclerosis discontinued brivaracetam due to abnormal behavior and eye movement disorder. The other TEAEs that led to discontinuation were decreased appetite (one patient), psychomotor hyperactivity/ sleep disorder (one patient), and convulsion (one patient). No deaths were reported in the trial. During the evaluation period, the percentage of patients/caregivers reporting TEAEs during each dose-escalation step was $37.4 \%, 35.4 \%$, and $27.4 \%$ for weeks 1, 2, and 3, respectively. Examination of individual TEAE data did not suggest a relationship with high plasma brivaracetam concentrations, although the numbers of patients were very limited.

Among patients with a history of focal seizures with or without secondary generalization and no primary generalized seizures aged 4 to $<16$ years, 23/34 (67.6\%) patients/ caregivers reported TEAEs (Table 2). The most common TEAE in this subgroup was psychomotor hyperactivity 
Table 1 Demographic and baseline epilepsy characteristics (safety set)

\begin{tabular}{|c|c|c|c|c|}
\hline \multirow[t]{2}{*}{ Characteristics } & \multicolumn{3}{|c|}{ Patients with focal seizures ${ }^{\mathrm{a}}$} & \multirow[t]{2}{*}{ Overall population $^{\mathrm{b}}(n=99)$} \\
\hline & Aged $<4$ years $(n=18)$ & Aged 4 to $<16$ years $(n=34)$ & Total $(n=52)$ & \\
\hline Age (years) & $1.3 \pm 0.7(0.3-2.8)$ & $9.0 \pm 4.0(4.1-15.6)$ & $6.3 \pm 4.9(0.3-15.6)$ & $6.3 \pm 4.8(0.2-15.6)$ \\
\hline Sex, female & $6(33.3)$ & $20(58.8)$ & $26(50.0)$ & $51(51.5)$ \\
\hline \multicolumn{5}{|l|}{ Race } \\
\hline White & $14(77.8)$ & $26(76.5)$ & $40(76.9)$ & 79 (79.8) \\
\hline Black & $1(5.6)$ & $3(8.8)$ & $4(7.7)$ & $4(4.0)$ \\
\hline Other & $3(16.7)$ & $5(14.7)$ & $8(15.4)$ & $16(16.2)$ \\
\hline Weight (kg) & $10.0 \pm 2.9$ & $32.8 \pm 14.6$ & $24.9 \pm 16.1$ & $24.2 \pm 16.2$ \\
\hline Age at epilepsy onset (years) & $0.4 \pm 0.5$ & $3.5 \pm 3.8$ & $2.4 \pm 3.4$ & $2.5 \pm 3.3$ \\
\hline Time since diagnosis (years) & $0.8 \pm 0.8$ & $5.5 \pm 4.0$ & $3.9 \pm 4.0$ & $3.8 \pm 3.7$ \\
\hline \multicolumn{5}{|l|}{ Epileptic syndrome $^{c}$} \\
\hline Localization-related & $14(77.8)$ & $33(97.1)$ & $47(90.4)$ & $51(51.5)$ \\
\hline Generalized & $1(5.6)$ & 0 & $1(1.9)$ & $40(40.4)$ \\
\hline Undetermined & $4(22.2)$ & 0 & $4(7.7)$ & $13(13.1)$ \\
\hline Special syndromes & 0 & $1(2.9)$ & $1(1.9)$ & $4(4.0)$ \\
\hline \multicolumn{5}{|c|}{$\begin{array}{l}\text { Seizure types experienced during } \\
\text { baseline }^{c}\end{array}$} \\
\hline Focal & $15(83.3)$ & $22(64.7)$ & $37(71.2)$ & $66(66.7)$ \\
\hline Generalized & 0 & 0 & 0 & $47(47.5)$ \\
\hline Unclassified & 0 & 0 & 0 & $6(6.1)$ \\
\hline Baseline seizure days ${ }^{\mathrm{d}}$ & $17.6 \pm 11.2$ & $9.4 \pm 10.8$ & $12.2 \pm 11.5$ & $15.5 \pm 11.2$ \\
\hline \multicolumn{5}{|l|}{ Number of prior AEDs } \\
\hline $0-1$ & $12(66.7)$ & $10(29.4)$ & $22(42.3)$ & $37(37.4)$ \\
\hline $2-4$ & $5(27.8)$ & $13(38.2)$ & $18(34.6)$ & $32(32.3)$ \\
\hline$\geq 5$ & $1(5.6)$ & $11(32.4)$ & $12(23.1)$ & $30(30.3)$ \\
\hline \multicolumn{5}{|l|}{ Number of concomitant AEDs } \\
\hline 1 & $10(55.6)$ & $10(29.4)$ & $20(38.5)$ & $32(32.3)$ \\
\hline 2 & $5(27.8)$ & $13(38.2)$ & $18(34.6)$ & $41(41.4)$ \\
\hline$\geq 3$ & $3(16.7)$ & $11(32.4)$ & $14(26.9)$ & $26(26.3)$ \\
\hline \multicolumn{5}{|l|}{ Concomitant $\mathrm{AEDs}^{\mathrm{e}}$} \\
\hline Valproate & $6(33.3)$ & $11(32.4)$ & $17(32.7)$ & $51(51.5)$ \\
\hline Topiramate & $4(22.2)$ & $12(35.3)$ & $16(30.8)$ & $27(27.3)$ \\
\hline Lamotrigine & 0 & $9(26.5)$ & $9(17.3)$ & $17(17.2)$ \\
\hline Clobazam & $3(16.7)$ & $3(8.8)$ & $6(11.5)$ & $14(14.1)$ \\
\hline Phenobarbital & $4(22.2)$ & $2(5.9)$ & $6(11.5)$ & $14(14.1)$ \\
\hline Oxcarbazepine & $3(16.7)$ & $7(20.6)$ & $10(19.2)$ & $13(13.1)$ \\
\hline
\end{tabular}

Data are presented as $n(\%)$ or mean \pm standard deviation (range) unless otherwise indicated $A E D$ antiepileptic drug

${ }^{\text {a }}$ Patients with a history of focal seizures with or without secondary generalization and no primary generalized seizures at baseline

${ }^{b}$ Patients with a history of any seizure type at baseline: focal, generalized, or unclassified

${ }^{\mathrm{c}}$ Patients could be included in more than one category

${ }^{\mathrm{d}}$ Number of seizure days, standardized to a 28-day duration; full analysis set

${ }^{\mathrm{e}}$ AEDs taken by $\geq 10 \%$ of patients overall

(4/34 patients [11.8\%]). Drug-related TEAEs were reported by $16 / 34(47.1 \%)$ patients/caregivers, most commonly psychomotor hyperactivity and somnolence (each 3/34 [8.8\%]). There were 2/34 (5.9\%) patients with serious TEAEs (both convulsion), one of which was considered by the investigator to be drug-related. Examination of individual TEAE data did not suggest a relationship with the use of specific comedications, although the numbers of patients were very limited.

Two patients had clinically relevant ECG changes during the evaluation period, which were not associated with 
Table 2 Summary of TEAEs and drug-related TEAEs reported during combined evaluation, downtitration, and safety (post-treatment) periods, and TEAEs during each week of the evaluation period (safety set)

\begin{tabular}{|c|c|c|c|c|}
\hline \multirow[t]{2}{*}{ TEAEs } & \multicolumn{3}{|c|}{ Patients with focal seizures ${ }^{\mathrm{a}}$} & \multirow{2}{*}{$\begin{array}{l}\text { Overall } \\
\text { population } \\
(n=99)\end{array}$} \\
\hline & Aged $<4$ years $(n=18)$ & $\begin{array}{l}\text { Aged } 4 \\
\text { to }<16 \text { years } \\
(n=34)\end{array}$ & Total $(n=52)$ & \\
\hline At least one TEAE & $16(88.9)$ & $23(67.6)$ & $39(75.0)$ & $66(66.7)$ \\
\hline Severe TEAEs & $2(11.1)$ & 0 & $2(3.8)$ & $3(3.0)$ \\
\hline Serious TEAEs & $4(22.2)$ & $2(5.9)$ & $6(11.5)$ & $8(8.1)$ \\
\hline Discontinuations due to TEAEs & $1(5.6)$ & $5(14.7)$ & $6(11.5)$ & $6(6.1)$ \\
\hline Deaths & 0 & 0 & 0 & 0 \\
\hline \multicolumn{5}{|c|}{ At least one TEAE in evaluation period } \\
\hline Week 1 (low dose) & $9 / 18(50.0)$ & $13 / 34(38.2)$ & $22 / 52(42.3)$ & $37 / 99(37.4)$ \\
\hline Week 2 (mid dose) & $10 / 17(58.8)$ & $13 / 32(40.6)$ & $23 / 49(46.9)$ & $34 / 96(35.4)$ \\
\hline Week 3 (high dose) & $7 / 17(41.2)$ & $9 / 31(29.0)$ & $16 / 48(33.3)$ & $26 / 95(27.4)$ \\
\hline \multicolumn{5}{|c|}{$\begin{array}{l}\text { TEAEs (MedDRA version } 15.0 \text { preferred term) reported by } \geq 5 \% \text { of all } \\
\text { patients }\end{array}$} \\
\hline Convulsion & $3(16.7)$ & $3(8.8)$ & $6(11.5)$ & $10(10.1)$ \\
\hline Irritability & $3(16.7)$ & $2(5.9)$ & $5(9.6)$ & $8(8.1)$ \\
\hline Pyrexia & $4(22.2)$ & $1(2.9)$ & $5(9.6)$ & $8(8.1)$ \\
\hline Somnolence & $1(5.6)$ & $3(8.8)$ & $4(7.7)$ & $8(8.1)$ \\
\hline Decreased appetite & $2(11.1)$ & $2(5.9)$ & $4(7.7)$ & $7(7.1)$ \\
\hline Fatigue & 0 & $2(5.9)$ & $2(3.8)$ & $5(5.1)$ \\
\hline Pharyngotonsillitis & $2(11.1)$ & $1(2.9)$ & $3(5.8)$ & $5(5.1)$ \\
\hline \multicolumn{5}{|l|}{ Drug-related ${ }^{\mathrm{c}}$ TEAEs } \\
\hline At least one drug-related TEAE & $6(33.3)$ & $16(47.1)$ & $22(42.3)$ & $32(32.3)$ \\
\hline Serious drug-related TEAEs & 0 & $1(2.9)$ & $1(1.9)$ & $1(1.0)$ \\
\hline \multicolumn{5}{|c|}{$\begin{array}{l}\text { Drug-related } \mathrm{d}^{\mathrm{b}} \text { TEAEs (MedDRA version } 15.0 \text { preferred term) reported } \\
\text { by } \geq 3 \% \text { of all patients }\end{array}$} \\
\hline Somnolence & $1(5.6)$ & $3(8.8)$ & $4(7.7)$ & $7(7.1)$ \\
\hline Decreased appetite & $2(11.1)$ & $2(5.9)$ & $4(7.7)$ & $6(6.1)$ \\
\hline Fatigue & 0 & $2(5.9)$ & $2(3.8)$ & $4(4.0)$ \\
\hline Psychomotor hyperactivity & 0 & $3(8.8)$ & $3(5.8)$ & $3(3.0)$ \\
\hline Aggression & $1(5.6)$ & $2(5.9)$ & $3(5.8)$ & $3(3.0)$ \\
\hline Irritability & 0 & $2(5.9)$ & $2(3.8)$ & $3(3.0)$ \\
\hline
\end{tabular}

Data are presented as $\mathrm{n}(\%)$ or $\mathrm{n} / \mathrm{n}(\%)$

MedDRA Medical Dictionary for Regulatory Activities, TEAE treatment-emergent adverse event

a Patients with a history of focal seizures with or without secondary generalization and no primary generalized seizures at baseline

${ }^{b}$ Patients with a history of any seizure type at baseline: focal, generalized, or unclassified

${ }^{c}$ According to the investigator or if relationship to treatment was not specified

any AE; one patient had ventricular hypertrophy and left axis deviation that returned to normal after 3 weeks, and the other had sinus bradycardia with possible left ventricular hypertrophy, but presented with a normal ECG 1 week later. Neither patient discontinued brivaracetam because of these events. An additional patient reported ventricular extrasystoles as a TEAE, which was not considered to be related to the trial drug, so the brivaracetam dose was not altered. No clinically meaningful changes in laboratory tests, body weight, or vital signs were observed.

\subsection{Plasma Concentration of Brivaracetam and Metabolites}

Trough plasma concentrations of brivaracetam and its metabolites increased proportionally with the brivaracetam dose, a finding that was consistent for each age group (Table 3). At the end of each week in the evaluation period, geometric mean trough concentrations of brivaracetam and its metabolites were marginally higher in the group aged 4 to $<16$ years compared with the group aged $<4$ years. 
Table 3 Trough plasma concentrations of brivaracetam and its metabolites during the evaluation period for patients with focal seizures (pharmacokinetic-per-protocol set)

\begin{tabular}{|c|c|c|c|}
\hline & \multicolumn{3}{|c|}{ Trough plasma concentration $(\mu \mathrm{g} / \mathrm{mL})^{\mathrm{a}}$} \\
\hline & \multicolumn{3}{|c|}{ Patients with focal seizures ${ }^{b}$} \\
\hline & Aged $<4$ years & Aged 4 to $<16$ years & Total \\
\hline End of week 1 (low dose) & $n=11$ & $n=19$ & $n=30$ \\
\hline Brivaracetam $^{c}$ & $0.201(64.4)$ & $0.279(81.0)$ & $0.247(76.3)$ \\
\hline BRV-OH & $0.050(78.8)$ & $0.061(44.7)$ & $0.057(58.0)$ \\
\hline BRV-AC & $0.008(94.4)$ & $0.015(77.3)$ & $0.012(89.9)$ \\
\hline BRV-OHAC & $0.006(43.1)$ & $0.008(46.7)$ & $0.007(48.4)$ \\
\hline End of week 2 (mid dose) & $n=9$ & $n=11$ & $n=20$ \\
\hline Brivaracetam $^{\mathrm{d}}$ & $0.348(62.3)$ & $0.379(58.3)$ & $0.365(58.5)$ \\
\hline BRV-OH & $0.100(63.1)$ & $0.123(55.3)$ & $0.112(58.3)$ \\
\hline BRV-AC & $0.016(84.9)$ & $0.020(66.2)$ & $0.018(73.7)$ \\
\hline BRV-OHAC & $0.012(34.9)$ & $0.014(37.6)$ & $0.013(36.1)$ \\
\hline End of week 3 (high dose) & $n=7$ & $n=11$ & $n=18$ \\
\hline Brivaracetam $^{\mathrm{e}}$ & $0.598(60.6)$ & $0.761(57.7)$ & $0.693(58.5)$ \\
\hline BRV-OH & $0.200(96.4)$ & $0.277(56.0)$ & $0.244(72.2)$ \\
\hline BRV-AC & 0.028 (77.9) & $0.048(68.9)$ & $0.039(77.6)$ \\
\hline BRV-OHAC & $0.019(38.8)$ & $0.029(43.6)$ & $0.024(46.5)$ \\
\hline
\end{tabular}

Data are presented as geometric mean (coefficient of variation \%). All reported p-values can only be interpreted in an exploratory manner, i.e., are nominal

$B R V$-AC acid metabolite of brivaracetam, $B R V-O H$ hydroxy metabolite of brivaracetam, BRV-OHAC hydroxy acid metabolite of brivaracetam

${ }^{a}$ Multiply by 4.713646 to convert concentrations from $\mu \mathrm{g} / \mathrm{mL}$ to $\mu \mathrm{mol} / \mathrm{L}$. Metabolite concentrations are expressed in brivaracetam equivalents and use the same conversion factor

${ }^{b}$ Patients with a history of focal seizures with or without secondary generalization and no primary generalized seizures at baseline

${ }^{\mathrm{c}}$ Wilcoxon rank-sum test for difference between groups aged $<4$ years and 4 to $<16$ years, $p=0.17$

${ }^{\mathrm{d}}$ Wilcoxon rank-sum test for difference between groups aged $<4$ years and 4 to $<16$ years, $p=0.60$

${ }^{\mathrm{e}}$ Wilcoxon rank-sum test for difference between groups aged $<4$ years and 4 to $<16$ years, $p=0.48$

Broad interpatient variability was observed in the concentrations of brivaracetam and its metabolites. However, the differences for brivaracetam had $p$ values of $>0.05$ (Wilcoxon rank-sum test, see Table 3 ). Individual plasma concentration versus time profiles for brivaracetam at the low (day 7), mid (day 14), and high (day 21) dose levels is shown in Fig. S2 in the ESM. In addition, the relationship between predose plasma brivaracetam concentration and $\mathrm{mg} / \mathrm{kg}$ dose in the overall population is illustrated in Fig. S3.

\subsection{Exploratory Efficacy}

Over the 3 -week evaluation period, $\geq 50 \%$ responder rates (all seizure types) were $21.3 \%$ for all patients $(n=80)$ and $29.7 \%$ for patients with a history of focal seizures with or without secondary generalization and no primary generalized seizures ( $n=37$ ) (Fig. 2). Among patients with a history of focal seizures with or without secondary generalization and no primary generalized seizures, $\geq 50 \%$ responder rates were $20.0 \%$ for patients aged $<4$ years $(n=15)$ and $36.4 \%$ for those aged 4 to $<16$ years $(n=22)$.

In the overall trial population, the median number of seizure days (all seizure types; standardized to a 28-day duration) was 18.7 days during the baseline period and 16.0 days during the evaluation period, a median reduction of $4.8 \%$.

The median reduction from baseline in number of seizure days (all seizure types) was $14.3 \%$ in patients with a history of focal seizures with or without secondary generalization and no primary generalized seizures, and $0.0 \%$ in patients with a history of primary generalized seizures (Table 4).

The overall seizure freedom rate was $14 / 97(14.4 \%)$. Complete seizure freedom was reported by $12 / 50(24.0 \%)$ patients with a history of focal seizures with or without secondary generalization and no primary generalized seizures, comprising $4 / 18(22.2 \%)$ patients aged $<4$ years and $8 / 32$ $(25.0 \%)$ aged 4 to $<16$ years. 


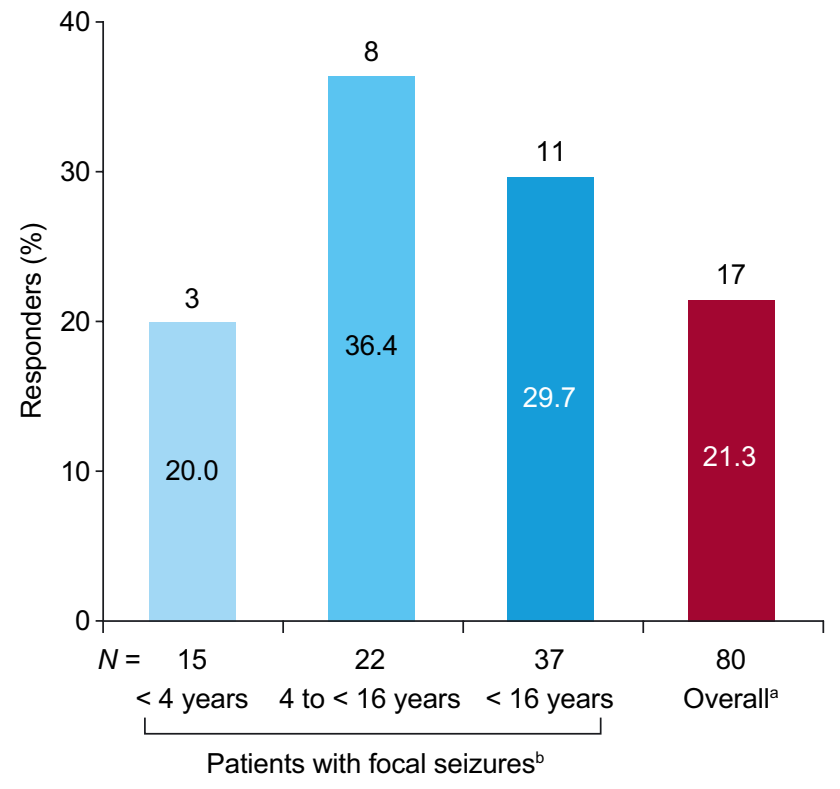

Fig. 2 Responder rate based on a $\geq 50 \%$ reduction in seizure days from baseline to the end of the evaluation period, according to seizure diary data overall and by age and seizure category (full analysis set

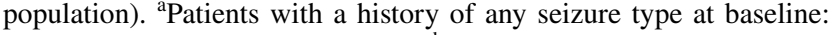
focal, generalized, or unclassified. ${ }^{b}$ Patients with a history of focal seizures with or without secondary generalization and no primary generalized seizures at baseline. Note: Patients with a zero seizure count during the baseline period were excluded from the analysis as percent change from baseline could not be calculated $(n=17)$. Numbers above bars represent the number of patients who responded to treatment

\section{Discussion}

This is the first trial to evaluate brivaracetam in infants, children, and adolescents with epilepsy. The results of this phase IIa open-label trial showed that the treatment of pediatric patients aged 1 month to $<16$ years with adjunctive brivaracetam oral solution was generally well tolerated. Since TEAEs related to common childhood infectious diseases are frequently reported in pediatric populations (in this study, primary system organ class 'infections and infestations': 26/99 [26.3\%]), drug-related TEAEs are often considered to be of greatest relevance in this population. The most commonly reported drug-related TEAEs were somnolence (7/99 [7.1\%]) and decreased appetite (6/99 [6.1\%]) in all patients, and psychomotor hyperactivity (3/34 [8.8\%]) and somnolence $(3 / 34[8.8 \%])$ in the subgroup aged 4 to $<16$ years with a history of focal seizures with or without secondary generalization and no primary generalized seizures. It should be noted that, because this trial was an open-label, 3-week, forced dose-escalation trial in infants and children with various epileptic syndromes (including focal seizures) and other symptomatic generalized epilepsies, the results cannot be directly compared with double-blind, placebo-controlled studies in adult patients with focal seizures only.

The current trial aimed to characterize the steady-state plasma concentrations of brivaracetam and its metabolites in pediatric patients. While the naïve pooled data analysis provided some evidence to suggest that trough plasma concentrations of brivaracetam may increase with age, non-linear mixed-effects modeling of the entire data set (i.e., also using non-trough plasma concentrations and demographic covariates) would be required to quantify the dose-concentration relationship among pediatric age groups. The related analysis was published elsewhere [13]. Furthermore, external validation using independent studies with large patient numbers would be necessary to confirm these observations. In this trial, brivaracetam oral solution was administered over 3 weeks in two equal divided daily doses of approximately $0.8,1.6$, and $3.2 \mathrm{mg} / \mathrm{kg} /$ day for patients aged $\geq 8$ years and $1.0,2.0$, and $4.0 \mathrm{mg} / \mathrm{kg} /$ day for patients aged $<8$ years. For each age group, trough brivaracetam and metabolite plasma concentrations increased proportionally with the weekly dose increases throughout the evaluation period, although high patient variability was observed and therefore no firm conclusions can be drawn. However, based on the data at the end of week 1 , where patient numbers were larger, the mean concentrations of brivaracetam (and its metabolites) only increased minimally between age groups, suggesting that the dosages used were appropriate, despite potential differences in renal maturation and expression of hepatic isoenzymes and obvious body size differences in these distinct groups. As a side note, the apparent modest increase in brivaracetam plasma concentration is consistent with a decrease in weightnormalized clearance with increasing child age [16]. Using this dosing regimen, no apparent increase in TEAEs was observed with increasing dose, which further supports the regimens used. The plasma concentrations of brivaracetam and demographic variables from this trial have been used to develop a population pharmacokinetic model of brivaracetam [13] that was used to derive pediatric dosing adaptation rules; these are reported in another publication [17].

The current trial provides preliminary evidence for the efficacy of adjunctive brivaracetam oral solution in pediatric patients. Among all patients who reported seizures during the baseline period $(n=80)$, the $\geq 50 \%$ responder rate was $21.3 \%$, and $14.4 \%$ of all patients $(n=97)$ reported complete seizure freedom during the evaluation period. Although patient numbers were small, there was also preliminary evidence of efficacy in the subgroup of patients with a history of focal seizures aged 4 to $<16$ years, with a $\geq 50 \%$ responder rate of $36.4 \%(n=22)$ and complete seizure freedom rate of $25.0 \%(n=32)$. Extrapolation of efficacy data from adults to predict treatment response is now accepted by the FDA in children with focal seizures aged $\geq 4$ years [14], 
Table 4 Number of seizure days (all seizure types) during baseline and evaluation periods and reduction from baseline in number of seizure days (full analysis set)

\begin{tabular}{|c|c|c|c|}
\hline & \multicolumn{3}{|c|}{ Patients with focal seizures ${ }^{\mathrm{a}}$} \\
\hline & Aged $<4$ years & Aged 4 to $<16$ years & Total \\
\hline & $n=18$ & $n=32$ & $n=50$ \\
\hline \multicolumn{4}{|l|}{ Number of seizure days, ${ }^{b}$ median } \\
\hline Baseline period & 21.7 & 4.3 & 8.9 \\
\hline Evaluation period & 17.8 & 3.4 & 6.3 \\
\hline Absolute reduction from baseline in number of seizure days, median & 3.6 & 0.0 & 0.2 \\
\hline \multirow[t]{4}{*}{ Percent reduction from baseline in number of seizure days, ${ }^{\mathrm{c}}$ median } & 18.2 & 3.8 & 14.3 \\
\hline & \multicolumn{3}{|c|}{ Patients with primary generalized seizures ${ }^{\mathrm{d}}$} \\
\hline & Aged $<4$ years & Aged 4 to $<16$ years & Total \\
\hline & $n=18$ & $n=27$ & $n=45$ \\
\hline \multicolumn{4}{|l|}{ Number of seizure days, ${ }^{b}$ median } \\
\hline Baseline period & 28.0 & 18.7 & 20.0 \\
\hline Evaluation period & 23.4 & 16.0 & 20.0 \\
\hline Absolute reduction from baseline in number of seizure days, median & 0.0 & 0.0 & 0.0 \\
\hline Percent reduction from baseline in number of seizure days, ${ }^{\mathrm{e}}$ median & 0.0 & 2.4 & 0.0 \\
\hline
\end{tabular}

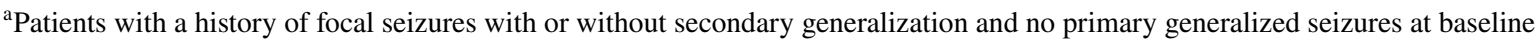

${ }^{\mathrm{b}}$ Number of seizure days, standardized to a 28-day duration

${ }^{c}$ Patients with a zero seizure count during the baseline period $(n=13)$ were excluded from the percent reduction analysis as percent change from baseline could not be calculated

${ }^{\mathrm{d}}$ Patients with a history of primary generalized seizures at baseline; patients could also have had focal seizures

${ }^{\text {e}}$ Patients with a zero seizure count during the baseline period $(n=4)$ were excluded from the percent reduction analysis as percent change from baseline could not be calculated

and similarly by the EMA for refractory focal seizures if the pediatric dose range is established [15].

Findings from this trial should be interpreted with the caveat that patient numbers in the subgroups were small, which makes it difficult to interpret the data in patients aged $<4$ years. Similarly, tolerability and efficacy could not be inferred in any specific epileptic syndrome as patient numbers were not high enough. In addition, brivaracetam treatment was open label, there was no control group, and brivaracetam was only administered for 3 weeks. Furthermore, this trial had no requirement for seizures to be reported during the baseline period. Therefore, efficacy evaluations that measured the change in seizure frequency from baseline could not be performed in patients without any seizures during the baseline period $(n=17)$.

\section{Conclusion}

This trial in children aged $<16$ years with epilepsy showed that short-term, adjunctive treatment with brivaracetam oral solution was generally well tolerated. Steady-state trough plasma concentrations of brivaracetam and its metabolites increased proportionally with the brivaracetam dose. The trial also provides preliminary evidence that adjunctive brivaracetam treatment is effective in pediatric patients.

Acknowledgements The authors thank the patients and their caregivers who contributed to this trial. They also thank the following people for their contributions to the trial: Esther Cardo Jalon, MD (Hospital Son Llatzer, Laboratorio de Neurociencias IUNICS, Palma de Mallorca, Baleares, Spain), Hugo Ceja Moreno, MD (Antiguo Hospital Civil de Guadalajara Fray, Guadalajara, Jalisco, Mexico), Bernard Dan, MD (Hopital Universitaire des Enfants Reine Fabiola, Brussels, Belgium), Francisco De la Pena, MD (Unidad de Investigación en Salud de Chihuahua, SC, Col. San Felipe, Chihuahua, Mexico), Anna Gniatkowska-Nowakowska, MD (Gabinet Lekarski Neurologii Dzieciecej i Leczenia Padaczki, Kielce, Swietokrzyskie, Poland), David Griesemer, MD (Tufts University School of Medicine, Boston, MA, USA), Helena Hojdikova, MD (Fakultni Necomcnice Hradec Kralove Neurology Clinic, Hradec Kralove, Czech Republic), Iwona Kochanowska, MD (EuroMedis Sp. z o.o., Szczecin, Zachodniopomorskie, Poland), Malgorzata Krause, MD (SPZOZ Dolnoslaski Szpital Specjalistyczny im. T Marciniaka, Centrum Medycyny Ratunkowej, Wroclaw, Dolnoslaskie, Poland), Lieven Lagae, MD (Universitair Ziekenhuis Leuven, Leuven, Belgium), Gustavo Lorenzo Sanz, MD (Hospital Universitario Ramon y Cajal, Madrid, Spain), Maria Mazurkiewicz-Beldzinska, MD (Uniwersyteckie Centrum Kliniczne, Gdansk, Pomorskie, Poland), Marie-Cecile Nassogne, MD (Cliniques Universitaires Saint Luc, Brussels, Belgium), Vilem Novak, DrSc (Fakultni Nemocnice Ostrava, Ostrava-Poruba, Czech Republic), Anup Patel, MD (Children's Hospital, Columbus, OH, USA), Barbara Prawdzic-Senkowska, MD (Wojewodzki Specjalistyczny Szpital Dzieciecy im. sw. Ludwika 
w Krakowie, Krakow, Malopolskie, Poland), James Benjamin Renfroe, MD (Child Neurology Center of Northwest Florida, PA, Gulf Breeze, FL, USA), Sarug Reyes Morales, MD (Instituto Biomedico de Investigacion, AC, Aguascalientes, Mexico), Frank Ritter, MD (Minnesota Epilepsy Group, PA, Saint Paul, MN, USA), Francesc Sanmarti, MD (Hospital San Joan de Deu, Barcelona, Spain), Wojciech Sobaniec, MD (Uniwersytecki Dzieciecy Szpital Kliniczny im. L Zamenhofa w Bialymstoku, Bialystok, Podlaskie, Poland), Barbara Steinborn, MD (Szpital Kliniczny im. Heliodora Swiecickiego Uniwersytetu Medycznego im. Karola Marcinkowskiego, Poznan, Wielkopolskie, Poland), Inna Vaisleib, MD (Children's Hospital of Pittsburgh, Pittsburgh, PA, USA), David Wang, MD (University of Rochester Medical Center, Rochester, NY, USA), and Angus Wilfong, MD (Texas Children's Hospital, Houston, TX, USA). The authors also acknowledge Barbara Pelgrims, $\mathrm{PhD}$ (UCB Pharma, Brussels, Belgium) for publication coordination. Medical writing support (assisting to develop the outline, first draft, and subsequent drafts, circulating for authors' review, and addressing comments) was provided by Rebecca Hurst, $\mathrm{PhD}$ (QXV Communications, an Ashfield Company, part of UDG Healthcare plc, Macclesfield, UK), funded by UCB Pharma. Additional editorial support (circulating later drafts for authors' review and addressing comments) was provided by Richard Fay, PhD, CMPP (Evidence Scientific Solutions, Philadelphia, PA, USA) and Emily Chu, PhD (Evidence Scientific Solutions, London, UK), funded by UCB Pharma.

Author contributions DD, BM, AS, and TD contributed to the conception and design of the trial. EL, DD, BM, and AS contributed to the acquisition of the data. All authors contributed to the analysis and interpretation of data, critically revised the manuscript, and gave final approval of the version to be submitted.

\section{Compliance with Ethical Standards}

Funding This work was funded by UCB Pharma. UCB Pharma was responsible for the design and conduct of the trial, the collection, analysis, and interpretation of data, and was involved in the preparation and review of the manuscript. The authors made the final decision to submit the manuscript for publication. Open access was funded by UCB Pharma.

Conflicts of interest Edwin Liu has no conflicts of interest that are directly relevant to the content of this article. Deanne Dilley was an employee of UCB Pharma when the trial was conducted and is currently employed by Statistics and Data Corporation. Belinda McDonough was an employee of UCB Pharma when the trial was conducted and is currently Director of MAFSAF Ltd. She has received stocks and stock options from UCB Pharma. Armel Stockis and Tony Daniels are employees of UCB Pharma. Armel Stockis has received stock options from UCB Pharma. Tony Daniels has received stocks from UCB Pharma.

Informed consent Informed consent was obtained from all individual participants included in the trial.

Ethical approval All procedures performed in studies involving human participants were in accordance with the ethical standards of the institutional and/or national research committee and with the 1964 Helsinki declaration and its later amendments or comparable ethical standards.

Data availability statement Due to the small sample size in this trial, individual patient data cannot be adequately anonymized and there is a reasonable likelihood that individual participants could be re-identified. For this reason, data from this trial cannot be shared.
Open Access This article is distributed under the terms of the Creative Commons Attribution-NonCommercial 4.0 International License (http://creativecommons.org/licenses/by-nc/4.0/), which permits any noncommercial use, distribution, and reproduction in any medium, provided you give appropriate credit to the original author(s) and the source, provide a link to the Creative Commons license, and indicate if changes were made.

\section{References}

1. Hwang H, Kim KJ. New antiepileptic drugs in pediatric epilepsy. Brain Dev. 2008;30(9):549-55.

2. Biton V, Berkovic SF, Abou-Khalil B, Sperling MR, Johnson ME, Lu S. Brivaracetam as adjunctive treatment for uncontrolled partial epilepsy in adults: a phase III randomized, double-blind, placebo-controlled trial. Epilepsia. 2014;55(1):57-66.

3. Klein P, Schiemann J, Sperling MR, Whitesides J, Liang W, Stalvey T, et al. A randomized, double-blind, placebo-controlled, multicenter, parallel-group study to evaluate the efficacy and safety of adjunctive brivaracetam in adult patients with uncontrolled partial-onset seizures. Epilepsia. 2015;56(12):1890-8.

4. Ryvlin P, Werhahn KJ, Blaszczyk B, Johnson ME, Lu S. Adjunctive brivaracetam in adults with uncontrolled focal epilepsy: results from a double-blind, randomized, placebo-controlled trial. Epilepsia. 2014;55(1):47-56.

5. UCB, Brussels, Belgium. Briviact ${ }^{\circledR}$ US Prescribing information. 2018. https://www.ucb.com/_up/ucb_com_products/documents/ Briviact_20180510_US-PI.pdf. Accessed 5 June 2019.

6. UCB, Brussels, Belgium. Summary of product characteristics Briviact $^{\circledR}$ (EU). https://www.ema.europa.eu/en/documents/ product-information/briviact-epar-product-information_en.pdf. Accessed 5 June 2019.

7. Rolan P, Sargentini-Maier ML, Pigeolet E, Stockis A. The pharmacokinetics, CNS pharmacodynamics and adverse event profile of brivaracetam after multiple increasing oral doses in healthy men. Br J Clin Pharmacol. 2008;66(1):71-5.

8. Sargentini-Maier ML, Espié P, Coquette A, Stockis A. Pharmacokinetics and metabolism of 14C-brivaracetam, a novel SV2A ligand, in healthy subjects. Drug Metab Dispos. 2008;36(1):36-45.

9. Stockis A, Hartstra J, Mollet M, Hadi S. Bioavailability and bioequivalence comparison of brivaracetam 10,50, 75, and $100 \mathrm{mg}$ tablets and $100 \mathrm{mg}$ intravenous bolus. Epilepsia. 2016;57(8):1288-93.

10. Stockis A, Watanabe S, Rouits E, Matsuguma K, Irie S. Brivaracetam single and multiple rising oral dose study in healthy Japanese participants: influence of CYP2C19 genotype. Drug Metab Pharmacokinet. 2014;29(5):394-9.

11. Nicolas JM, Chanteux H, Rosa M, Watanabe S, Stockis A. Effect of gemfibrozil on the metabolism of brivaracetam in vitro and in human subjects. Drug Metab Dispos. 2012;40(8):1466-72.

12. Otoul C, Watanabe S, McCabe S, Stockis A. Relative bioavailability and bioequivalence of brivaracetam $10 \mathrm{mg} / \mathrm{mL}$ oral solution and 50-mg film-coated tablet. Clin Pharmacol Drug Dev. 2017;6(3):313-7.

13. Schoemaker R, Wade JR, Stockis A. Brivaracetam population pharmacokinetics in children with epilepsy aged 1 month to 16 years. Eur J Clin Pharmacol. 2017;73(6):727-33.

14. No authors. Pediatric news. J Pediatr Pharmacol Ther. 2016;21(1):98.

15. European Medicines Agency. Guideline on clinical investigation of medicinal products in the treatment of epileptic disorders. 2010. http://www.ema.europa.eu/docs/en_GB/document_library/Scien 
tific_guideline/2010/01/WC500070043.pdf. Accessed 12 Jan 2018.

16. Anderson GD. Developmental pharmacokinetics. Semin Pediatr Neurol. 2010;17(4):208-13.
17. Schoemaker R, Wade JR, Stockis A. Extrapolation of a brivaracetam exposure-response model from adults to children with focal seizures. Clin Pharmacokinet. 2018;57(7):843-54. 